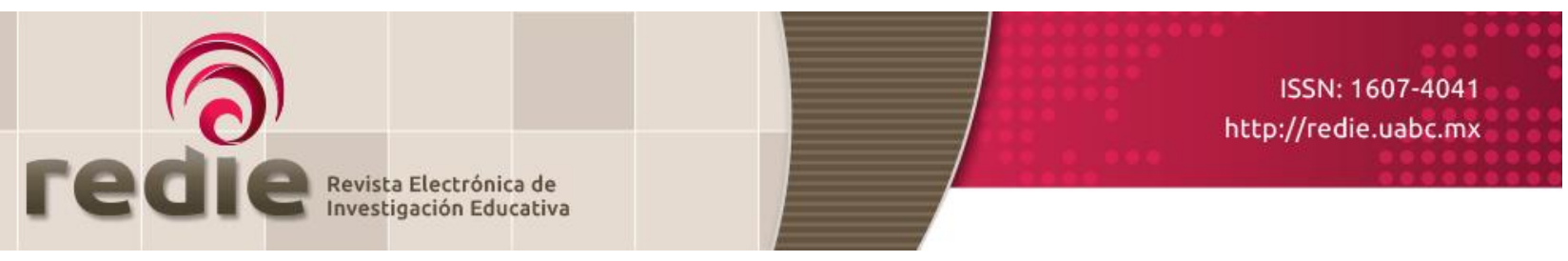

Vol. 20, Núm. 2, 2018

\title{
Autoetnografía y directivos docentes: una aproximación experiencial a las reformas educativas en Chile ${ }^{1}$
}

\section{Autoethnography and School Principals: an Experiential Approach to Educational Reforms in Chile}

\author{
Felipe Aravena Castillo (*) felipe.aravena@pucv.cl \\ Marta Quiroga Lobos (*) marta.quiroga@pucv.cl \\ (*) Pontificia Universidad Católica de Valparaíso \\ (Recibido: 23 de septiembre de 2016; Aceptado para su publicación: 3 de enero de 2017)
}

Cómo citar: Aravena, F. y Quiroga, M. (2018). Autoetnografía y directivos docentes: una aproximación experiencial a las reformas educativas en Chile. Revista Electrónica de Investigación Educativa, 20(2), 113-125. https://doi.org/10.24320/redie.2018.20.2.1600

\section{Resumen}

En los últimos 26 años se han implementado en Chile numerosas reformas educativas con el objetivo de mejorar la equidad y calidad del sistema. Los directivos escolares, como actores y protagonistas de estas reformas, han desarrollado su propia visión sobre estos fenómenos sociales y sus efectos en las organizaciones escolares, por ello el presente estudio tiene por objetivo analizar tres políticas públicas educativas a través de la experiencia de directivos docentes senior -utilizando una exploración autoetnográfica que se inscribe en el paradigma cualitativo. Los resultados indican que los directivos docentes vivencian las políticas públicas con temor y sensación de inseguridad percibida desde una posición solitaria. La relevancia de este estudio se enmarca en la oportunidad de situar a los individuos, desde una perspectiva emocional, en un contexto sociocultural específico.

Palabras clave: Autoetnografía, directores escolares, desarrollo profesional docente, reformas educativas.

\section{Abstract}

In the last 26 years, many educational reforms have been introduced in Chile with the aim of improving equity and quality in the system. School principals, as both actors and protagonists of these reforms, have developed their own vision of these social phenomena and their effects on educational organizations. It is on this basis that this study seeks to analyze three public policies in education through the experience of senior school principals, using an autoethnographic exploration that is part of a qualitative paradigm. Results show that school principals face these public policies with feelings of fear and uncertainty, and a perceived sense of isolation. The relevance of this study resides in the opportunity to position individuals, from an emotional perspective, in a specific sociocultural context.

Keywords: Autoetnography, school principals, teacher professional development, educational reforms.

\footnotetext{
${ }^{1}$ Este proyecto contó con el patrocinio de la Escuela de Pedagogía de la Pontificia Universidad Católica de Valparaíso.
} 


\section{Introducción}

La autoetnografía es un método cualitativo que ha emergido dentro del posmodernismo filosófico (Anderson, 2006; Chang, 2008; Wall, 2006). Este método se basa en una escritura altamente personalizada que estimula la reedición de emociones e historias en un contexto sociocultural claramente delimitado (Curran, 2012; Chang, Longman y Franco, 2014; Holt, 2003; Humphreys, 2005; Wall, 2006). Desde allí, el individuo realiza un recorrido en un espacio y tiempo concreto, delimitado por un evento crítico que produce una revisión sobre el pasado. Así, la autoetnografía permite reflejar lo vivenciado en una narración autogenerada, que posibilita la generación de aprendizaje y crecimiento personal basado en experiencias.

El presente estudio de caso múltiple tuvo por objetivo analizar tres políticas públicas educativas a través de la experiencia de siete directivos docentes senior en el contexto chileno. Para ello se utilizó una exploración autoetnográfica, que se inscribe en el paradigma cualitativo. La relevancia de este estudio se enmarca en la oportunidad de situar a los individuos, desde una perspectiva emocional, en un contexto sociocultural específico. Así se apuesta por analizar y repensar las fortalezas, desafíos y problemáticas en el mundo educativo a través del reposicionamiento del individuo en las organizaciones escolares y la política pública. A un nivel de los big data y las macro-reformas, los individuos y su quehacer tienden a homogeneizarse. En dicha aproximación se pierde el sentido de individualidad y emocionalidad, clave en todo proceso de aprendizaje. El significado y utilidad de lo aprendido en la escuela se sitúa a un nivel individual y colectivo; sin embargo, si la mirada sólo se reduce dirigiendo la atención investigativa en macroprocesos y estructuras, la invisibilización de los individuos emergerá. Esta invisibilización emergente es un riesgo que amenaza la educación porque tiende a estandarizar los procesos, olvidando el accionar de los estudiantes, profesores y directivos docentes. De manera que este estudio buscó mostrar la voz de los protagonistas.

El aprendizaje por descubrimiento, o mejor dicho por autodescubrimiento, es una posibilidad que abre la autoetnografía como método (Chang et al., 2014). El aprendizaje se genera cuando los individuos se descubren a sí mismos como protagonistas de su propia historia, ya que si bien son parte de un medio sociocultural compartido, la interacción con ese medio queda contenida en un espiral de procesos individuales. Por lo tanto, es un aprendizaje que transita en una dimensión dual: por un lado, una dimensión más centrada en procesos de aprendizaje a nivel personal y, por otro, en procesos de aprendizaje colectivos. Ellis (2009) agrega una segunda dualidad cuando se realiza una autoetnografía, subrayando la importancia de mezclar corazón y mente, ya que aquello permite aumentar las posibilidades de capitalizar aprendizajes profundos y duraderos. Por ello, la autoetnografía se presenta como un mundo de dualidades que sitúa a la persona entre las emociones y la racionalidad, entre lo individual y colectivo, entre lo privado y lo público.

Siguiendo la misma línea de pensamiento, otra dualidad que se desencadena es en la categorización de autoetnografía, situándola entre ciencia y arte. Chang (2008) señala que es una ciencia, porque es un método que indica cómo se puede realizar una aproximación al sujeto estudiado, cómo recolectar datos, analizarlos e interpretarlos, entre otros elementos propios del método. Sin embargo, Ellis y Bochner (2000) presentan una visión más sumida en la emocionalidad del narrador, es decir, en el arte. Esta apuesta, sin desconocer lo anterior en términos de método, explica que la autoetnografía es un arte porque el que un individuo estructure su emocionalidad sobre su propio recorrido sociocultural es algo difícil, sino es que imposible de realizar.

En función de lo anterior, se desprende la constante -y muy relevante crítica- que hace referencia a la calidad y validez de la autoetnografía como método cualitativo. Se tiende a pensar que la autoetnografía es poco confiable porque presenta elementos personales naturalmente subjetivos que obstaculizan la objetivación. Para Blinne (2014) las autoetnografías han sido criticadas a causa de la carencia de objetividad en el método, evidenciada en una falta de credibilidad y validez investigativa. Wolcott (2008) agrega que esta falta de credibilidad y validez se vislumbra porque la etnografía tiende a interactuar con el medio directamente a través de los actores sociales. En contraste, en la autoetnografía el investigador queda situado en un historical record subjetivo, profundamente personal y con foco en las emociones. Esto plantea una discusión central en el rol del etnógrafo (Blinne, 2014; Ellis y Bochner, 2000; Wolcott; 2008) en una dimensión que complejiza la relación entre lo subjetivo y objetivo. 
En la autoetnografía el rol del investigador-narrador es vital porque es el sujeto quien interactúa de manera experiencial con los procesos socioculturales en los cuales se encuentra inmerso. El individuo, considerando la autoetnografía como método cualitativo, pasa a ser un investigador-narrador (Wall, 2006). Esto es importante porque sitúa a la persona en un rol de analista crítico, obligando al individuo a realizar procesos de auto-análisis, auto-introspección y auto-observación (Ellis y Bochner, 2000; Wolcott, 2008; Chang, 2008; Ellis, 2009) enfatizan que este enfoque más analítico no tiene sentido si no se piensa la narración desde un prisma sociocultural. En este sentido, el énfasis sociocultural marca una diferencia cualitativa entre historias de vida, autobiografía y memorias, puesto que exige que el individuo se enfrente a tensiones culturales que son propias del campo de acción de la etnografía, como finalidad y método; en la etnografía el etnógrafo no tiende a analizarse a sí mismo, sino a la población que lo rodea. Sin embargo, en una narración personal el investigador y el investigado son lo mismo, investigador y narrador son inseparables. En otras palabras, la autoetnografía utiliza al mismo investigador como sujeto de estudio, siendo el primer participante.

\subsection{Autoetnografía y procesos educativos}

Durante los últimos 10 años, la autoetnografía como método ha sido conectada con el campo educativo (Austin y Hickey, 2007; Reed-Denahay, 2009; Vasconcelos, 2011). La escuela es una institución que se encuadra en un marco sociocultural presentando relaciones de poder y conflicto; lo anterior aumenta la pertinencia de la autoetnografía como método de análisis, observación e interpretación personal para promover la construcción constante de un ser reflexivo en situaciones conflictivas. Para Legee (2014) y Chang et al. (2014), la autoetnografía es clave para potenciar las experiencias de vida: la tarea de aprender y enseñar es fundamentalmente una experiencia de vida que interactúa con un medio cultural de manera constante. La autoetnografía presenta utilidad en el mundo educativo porque entrega la oportunidad de entender los cambios de manera sistémica, las prácticas realizadas, las prácticas no realizadas y las emociones a través de experiencias de vida relatadas por los mismos profesores, estudiantes y directivos.

Day y Gu (2012) han demostrado que los cambios en las políticas públicas afectan la vida profesional y personal de los profesores, por ello es importante estudiar retrospectivamente, a través de sus voces, "cómo se vivió" en su contexto la implementación de determinadas políticas públicas. No sólo es importante centrar la mirada en los efectos de las políticas públicas a un nivel estructural y sistémico, sino mirar lo experimentado por los protagonistas de los cambios. De ahí que para este estudio se seleccionaron tres políticas públicas, que fueron consciente e intencionalmente seleccionadas porque se caracterizaron por generar una discusión nacional que involucró a diferentes actores sociales, incorporando nuevos recursos financieros y provocando un cambio estructural y cultural dentro y fuera de las organizaciones escolares. Es así como la autoetnografía, desde un enfoque personal y vivenciado, potencia el análisis de estas macropolíticas públicas, reposicionando a los individuos que escriben su historia.

\subsection{Principales reformas educativas en Chile}

En los últimos 26 años el sistema educativo en Chile ha estado en constante cambio, en este proceso se distinguen cinco fases:

1) Mejora de condiciones para la docencia (1990-1994). Finalizada la dictadura, una de las principales preocupaciones fue recuperar los salarios de los profesores y condiciones de trabajo, para ello se creó el Sistema Nacional de Evaluación de Desempeño (SNED), premio a la excelencia académica de los establecimientos que se traduce en recursos financieros adicionales al sueldo de los profesores y directivos. Se instalan programas focalizados: P-900, MECE Básica y Programa Enlaces. Se aumentan los recursos privados en el sistema público a través de la ley de donaciones de las empresas con fines educacionales (1993) y se crea el financiamiento compartido (modificación al DFL [Decreto con Fuerza de Ley] 5/92) (Ministerio de Educación [Mineduc], 2008), cuyo fin era inyectar recursos a la educación por parte de los padres y no del estado.

2) Tiempo para aprender y cambios en el currículum (1994-2000). Durante este período los principales cambios se asocian a la promulgación del Estatuto Docente Ley 19.070 (Mineduc, 2008), que regula la profesión docente. En 1995 se crea la Bonificación Proporcional y Planilla Complementaria (SAE), Ley 
19.410. En 1997 se dicta la Ley 19.352, que instala la Jornada Escolar Completa (JEC). En 1998 se establece el Decreto 220: nuevo currículum en educación básica y media. Se desarrollan políticas de cobertura de textos escolares y se promueve la incorporación de las TIC al proceso de enseñanza y aprendizaje. Se aumentan los recursos para los MECE Básica-Media.

3) Evaluación Docente y Directiva (2000-2006). En este período se extiende la Educación Obligatoria gratuita a 12 años. En 2004 se instalan la Ley 19.961 de Evaluación Docente Municipal, la Ley 19.979 de Desempeño Directivo y la Ley 19.933 de Asignación de Desempeño Colectivo. Se establecen incentivos para desempeño de los profesionales de la educación: Asignación de Excelencia Pedagógica (AEP), Bonificación de Reconocimiento Profesional (BRP) y Bonificación de Reconocimiento Profesional (AVDI).

4) Focalización en la calidad educativa (2006-2012). En 2008 se promulga la Ley 20.248, Ley de Subvención Escolar Preferencial (SEP), que paulatinamente se extiende hasta enseñanza media. En el 2009 se promulga la Ley General de Educación (LGE), Ley 20.370. En el 2011 se promulga la Ley de Calidad y Equidad de la Educación, Ley 20.501, y la Ley de Sistema Nacional de Aseguramiento de la Calidad de la Educación Básica y Media y su Fiscalización, Ley 20.529. En el 2012 se pone en marcha la Agencia de Calidad y se inicia la Superintendencia de Educación en Chile.

5) Focalización en la equidad (2012 a 2016). Recientemente se aprobó la Ley 20.903, que crea el Sistema de desarrollo profesional docente, y se promulga la Ley 20.845 de inclusión escolar, que regula la admisión de los estudiantes, elimina el financiamiento compartido y prohíbe el lucro en establecimientos educativos que reciben aportes del estado.

Como se puede observar, en los últimos 26 años el sistema educativo ha cambiado sustancialmente, siendo los docentes y directivos docentes del país actores privilegiados. Рara este estudio se han seleccionado tres políticas públicas que cumplen con los siguientes criterios: alto interés de diferentes actores sociales en su proceso legislativo, alto impacto en las organizaciones e individuos con su implementación, e inyección de recursos financieros adicionales al sistema escolar por parte del Estado para su implementación. Las políticas seleccionadas bajo estos criterios son: Evaluación Docente, con alto impacto en el desarrollo profesional de los docentes; Ley de Subvención Preferencial 2008, con alto impacto en las organizaciones escolares; y Ley de Alta Dirección Pública, con alto impacto en el liderazgo y gestión escolar, que regula el acceso al cargo de director en escuelas municipales.

a) Evaluación Docente. Actualmente se implementan tres sistemas de evaluación docente. Cada uno con características particulares que responden a los criterios del Marco de la Buena Enseñanza y tienen un impacto en la carrera docente y en las remuneraciones. Es importante mencionar que los docentes que trabajan para el sistema municipal están obligados a realizar la evaluación docente. En cambio, quienes trabajan en establecimientos particulares subvencionados son voluntarios y pueden acceder al sistema de excelencia pedagógica. A continuación se explican brevemente:

1) En 2002 se creó el sistema de Asignación de Excelencia Pedagógica (AEP), el cual es voluntario e individual para los profesores que trabajan en establecimientos subvencionados. Ellos deben elaborar un portafolio que incluye una planificación de clases, estrategias de evaluación de alumnos, filmación de una clase y reflexión sobre su práctica pedagógica. Una vez evaluado el portafolio, los docentes deben aprobar una prueba de selección múltiple sobre conocimientos disciplinares y pedagógicos de acuerdo al nivel de enseñanza y disciplina en que se desempeñan habitualmente. Al año, el $30 \%$ de los postulantes tiene resultados exitosos, aprobar esta evaluación significa un aumento del $8 \%$ de la remuneración anual. Los docentes también son invitados a participar en la Red de Maestros.

2) En 2004 entró en vigencia la Evaluación Docente (Ley N¹9.961), proceso obligatorio para los docentes que trabajan en el sistema municipal, debiendo evaluarse cada cuatro años. El Ministerio de Educación define anualmente los sectores de aprendizajes y niveles en que se realizará la evaluación, que es coordinada administrativamente por un funcionario del municipio correspondiente. El proceso de evaluación consta de cuatro insumos: el portafolio que elabora el profesor (en el que se incluye la planificación, evaluación, filmación de 45 minutos de una clase previamente seleccionada por el profesor y reflexión sobre una unidad), equivalente al $60 \%$ del resultado final, a lo que se suma una autoevaluación (10\%), un informe de terceros, director o jefe de la Unidad Técnica Pedagógica (UTP) (10\%) y una entrevista con un par evaluador (20\%). La 
implementación de esta política estuvo rodeada de controversias, más aún cuando el resultado del portafolio ha dado origen al encasillamiento en diferentes niveles para la carrera docente, proceso que actualmente se encuentra en ejecución. El resultado de la evaluación del profesor se expresa en cuatro niveles: insatisfactorio, básico, competente y avanzado. Los docentes con resultados insatisfactorios deben presentar al año inmediato siguiente la evaluación. De no cambiar los resultados, se han implementado políticas de desvinculación. Para los docentes evaluados como competentes o destacados existe la posibilidad de que voluntariamente se presenten al sistema de evaluación de Asignación Variable de Desempeño Individual (AVDI).

3) El sistema de Asignación Variable de Desempeño Individual (AVDI) es voluntario para los docentes de escuelas municipales con buenos resultados en la evaluación docente, quienes adicionalmente deben rendir la prueba de conocimientos disciplinarios y pedagógicos. De obtener buenos resultados, su remuneración recibe un incremento del 10\% anual.

Como se observa, el sistema de evaluación en cualquiera de sus tres modalidades tiene como requisito la elaboración de un portafolio, y a partir de los resultados de éste es factible rendir las evaluaciones correspondientes. Los resultados de los procesos voluntarios implican en ambos casos un aumento de sueldo.

Una de las fortalezas de los tres sistemas de evaluación docente es que está basado en un estándar fijado en el marco de la Buena Enseñanza, lo que facilita la identificación de brechas de desarrollo y la retroalimentación (Sun, Correa, Zapata y Carrasco, 2011).

El estudio realizado por Bravo, Falck, González, Manzi y Peirano (2008) evidencia que los "tres programas de certificación son exitosos en identificar a los docentes más efectivos entre quienes postulan o participan. Los docentes certificados o bien evaluados permiten que sus alumnos tengan los mejores resultados en las pruebas estandarizadas" (p. 21).

b) Ley de Subvención Preferencial (20.248) o Ley SEP. Se promulgó en el 2008. En ella se establece que los alumnos clasificados como vulnerables o prioritarios requieren de más recursos financieros y apoyo рага lograr aprendizajes de calidad, y que los establecimientos que los atienden deben realizar esfuerzos sistemáticos y planificados para el mejoramiento de la calidad de los aprendizajes. Por ello los establecimientos educacionales deben elaborar un plan de mejora a partir de un diagnóstico institucional que considere la planificación de las metas anuales, la implementación de las acciones planificadas, el seguimiento de los logros y la rendición de cuentas por los resultados obtenidos. Se crea en paralelo un sistema de clasificación de escuelas y un sistema de apoyo, orientación y monitoreo que está a cargo de una institución: la Agencia de la Calidad. Eso implica un cambio en la estructura del sistema educativo. Según Raczynski, Muñoz, Weinstein y Pascal (2016, p.186), esta ley ha modificado la manera en que escuelas y sostenedores viven, proyectan y visualizan la mejora escolar y su responsabilidad en ésta, al tiempo que ha generado cambios en la gestión a nivel de directivos y docentes; sin embargo, se trata de un proceso en curso, todavía inmaduro, en parte porque sigue conviviendo con otras políticas y porque, como indica la evidencia internacional, los procesos de reforma y cambio educativo como éstos toman tiempo y no son lineales.

Se debe observar también que desde el 2014 la Agencia de la Calidad se ha ocupado de dar orientaciones, evaluar y apoyar a los establecimientos y realizar una difusión menos estigmatizadora de las escuelas con bajos resultados SIMCE y niveles de desempeño bajo. Esto ha permitido que los equipos directivos se sientan menos estigmatizados, sobre todo los que trabajan con alumnos vulnerables. Uno de los aspectos que más preocupa a los directivos docentes es el excesivo trabajo administrativo que demandan los procesos de rendición de cuentas, especialmente el financiero. Paulatinamente se evidencia más preocupación por la instalación de sistemas de monitoreo que permitan reconocer la efectividad de las acciones realizadas.

c) Alta Dirección Pública. La Ley 20.501 (2011) estableció que los directores de escuelas municipales deberán ser seleccionados por el sistema de Alta Dirección Pública. Se especifica la creación de una comisión calificadora integrada por el Jefe de Departamento de Administración de Educación Municipal (DAEM), un miembro del Consejo de Alta Dirección Pública y un docente de la dotación municipal. Los requisitos legales para postular al cargo son: ser ciudadano chileno mayor de 18 años y no haber cumplido ninguna pena aflictiva, poseer una salud compatible con el cargo, poseer el título de docente en 
universidades que presenten carreras pedagógicas de ocho a diez semestres, tener el grado de magíster en educación o cursos de perfeccionamiento de tipo administrativos y curriculares, y haber trabajado durante tres años continuos en un establecimiento educacional. Se valora la experiencia. El procedimiento indica que después de un análisis curricular de los postulantes, quienes cumplan con los requisitos serán sometidos a una entrevista psicolaboral. De estos resultados se desprende una lista de candidatos idóneos que son entrevistados por la comisión, quien elabora una terna que se presenta al alcalde de la comuna y éste puede seleccionar a cualquiera de los candidatos.

Los directivos, una vez seleccionados para el cargo, deben firmar un convenio de desempeño comprometiéndose a cumplir ciertas metas. El cargo es concursado por cinco años, transcurrido este período puede postular sólo por una vez más dentro del mismo establecimiento. En los casos que no se logren las metas comprometidas el director puede ser desvinculado.

\section{Método}

Esta investigación se desarrolla con la participación de siete directivos docentes con más de 13 años de experiencia en cargos directivos, que durante 9 meses participaron en un programa de formación de directivos docentes que tenía por objetivo actualizar sus conocimientos técnicos y promover el cambio en las prácticas de liderazgo escolar definidas en el Marco de la Buena Dirección y Liderazgo Escolar.

Los participantes en el curso tenían un promedio de 55 años. Todos ellos realizaron sus estudios de Pedagogía y primeros años de docencia durante la dictadura militar. Del análisis de sus ciclos de vida profesional, cuatro de ellos estudiaron Pedagogía como su primera opción, dos como una segunda opción y uno por su formación sacerdotal en una orden con vocación educativa.

La tabla I muestra la diversidad de los participantes, especialmente si se considera el tipo de dependencia y escuela.

Tabla I. Datos directivos docentes

\begin{tabular}{|c|c|c|c|c|c|c|c|}
\hline & Cargo & $\begin{array}{l}\text { Años como } \\
\text { directivo }\end{array}$ & $\begin{array}{l}\text { Años en la } \\
\text { institución }\end{array}$ & Género & $\begin{array}{l}\text { Tipo de } \\
\text { escuela }\end{array}$ & Sostenedor & $\begin{array}{l}\text { Niveles } \\
\text { que atiende }\end{array}$ \\
\hline 1 & Director & 12 & 6 & Masculino & $\begin{array}{l}\text { Particular } \\
\text { subvencionado }\end{array}$ & $\begin{array}{l}\text { Comunidad } \\
\text { religiosa }\end{array}$ & $\begin{array}{l}\text { Técnico } \\
\text { profesional }\end{array}$ \\
\hline 2 & Directora & 10 & 10 & Femenino & Municipal & Municipio & Escuela básica \\
\hline 3 & Directora & 15 & 1 & Femenino & Municipal & Municipio & Escuela básica \\
\hline 4 & Directora & 10 & 1 & Femenino & Municipal & Municipio & Escuela especial \\
\hline 5 & Inspector & 22 & 22 & Masculino & Municipal & Municipio & Adultos \\
\hline 6 & Inspector & 15 & 20 & Masculino & $\begin{array}{l}\text { Particular } \\
\text { subvencionado }\end{array}$ & $\begin{array}{l}\text { Comunidad } \\
\text { religiosa }\end{array}$ & $\begin{array}{l}\text { Colegio con } \\
\text { básica y media }\end{array}$ \\
\hline 7 & Inspector & 20 & 35 & Femenino & $\begin{array}{l}\text { Particular } \\
\text { subvencionado }\end{array}$ & $\begin{array}{l}\text { Comunidad } \\
\text { religiosa }\end{array}$ & $\begin{array}{l}\text { Colegio con } \\
\text { básica y media }\end{array}$ \\
\hline
\end{tabular}

Como se evidencia, los participantes tienen experiencia liderando organizaciones y por sus cargos les ha correspondido implementar algunas de las políticas comentadas en párrafos anteriores o saben sobre su implementación e impacto. Tres de ellos se desempeñan como Inspectores Generales, tienen a su cargo cautelar la sana convivencia de los alumnos y colaborar con los docentes en la resolución de conflictos; en otros momentos de sus vidas profesionales han ocupado otros cargos, como jefe de UTP y director subrogante.

La metodología utilizada consistió en un estudio cualitativo de casos múltiples, de carácter descriptivo, obteniendo por propósito indagar la experiencia de directivos docentes. Según Hamilton y CorbettWhittier (2012), los estudios de casos sirven fundamentalmente para comprender contextos, comunidades e individuos, pues capturan la esencia de los problemas, la complejidad de los contextos y las particularidades que los rodean (Maxwell, 2005). En la metodología de estudios de casos existen tres tipos: explicativo, exploratorio y descriptivo (Patton, 2001). El presente estudio opta por ser descriptivo porque busca recoger, agrupar y analizar datos relevantes para levantar categorías y conclusiones generales aplicables a un supra-nivel sin considerar una aproximación de hipótesis causales (Maxwell, 2005). Es decir, los datos emergentes permiten comprender el fenómeno y no al revés (Patton, 2001). 
Esto va en línea con la etnografía, puesto que los contextos explican la emergencia de la realidad vivida (Chang et al. 2014).

Las investigaciones autoetnográficas utilizan la escritura como el medio más adecuado para reflexionar sobre la acción profesional. Tal como afirma Polkinghorne (1995), la narración es "la única forma lingüística adecuada para mostrar la existencia humana como acción contextualizada" (p. 5), lo que permite comprender los significados con los que las personas componemos el mundo y expresamos la visión que tenemos de él (Bruner, 1997).

Las actividades de escritura realizadas en esta investigación son tres: la primera corresponde al ciclo de vida profesional, la segunda es una bitácora y la tercera es el taller de escritura autoetnográfica.

1. Elaboración de ciclos de vida profesional. Se solicitó a cada participante elaborar un relato escrito y un diagrama de su vida profesional utilizando como marco referencial la propuesta metodológica desarrollada por Day y Gu (2010), quienes proponen una metodología para representar los ciclos de desarrollo profesional docente que consiste en identificar los incidentes críticos que han sido un estímulo para su motivación, compromiso y autoeficacia en la trayectoria profesional (Flanagan, 1954). A medida que cada participante desarrollaba su relato se brindó retroalimentación sobre la estructura y el contenido del trabajo, para luego desarrollar un diagrama individual. Se obtuvo un relato, un diagrama y una metáfora de cada trayectoria. Esta actividad se realizó durante el tercer mes de formación. En los primeros dos meses los participantes analizaron y compartieron datos y caracterizaciones de las escuelas, lo que permitió ganar confianza entre ellos.

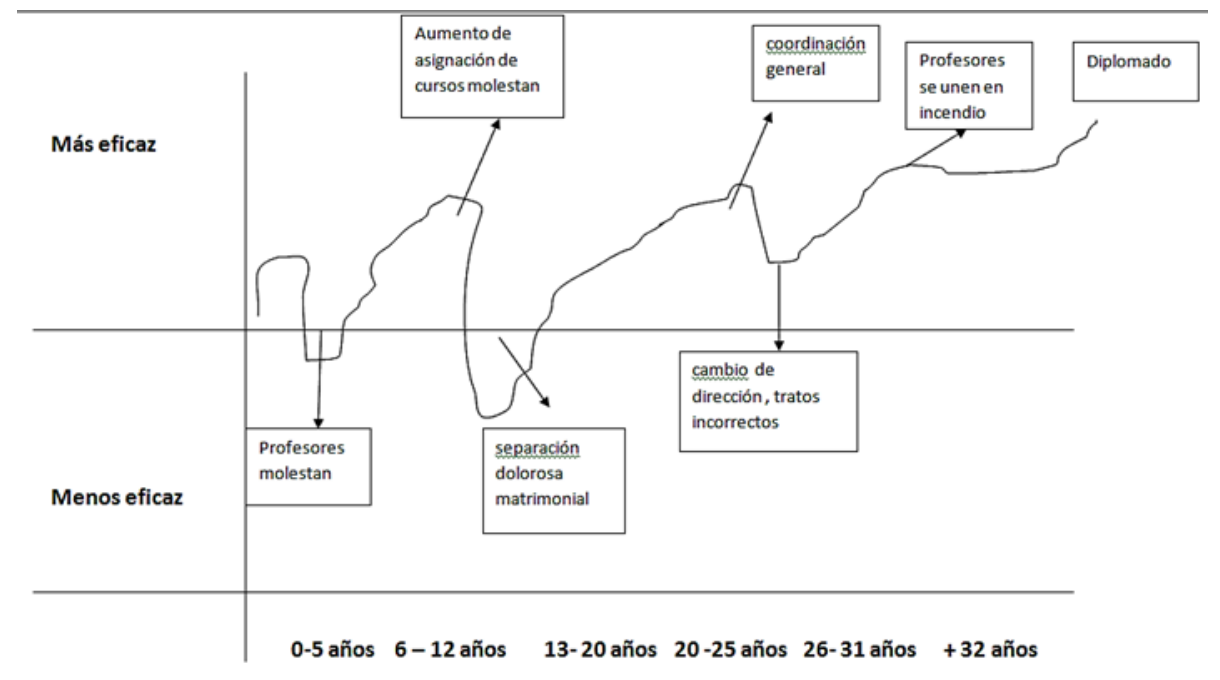

Figura 1. Diagrama del ciclo de vida profesional de los participantes

2. Bitácora de reflexión personal. Es un cuaderno personal que desarrolla cada participante, en el cual registran sus pensamientos a medida que se desarrolla el proceso formativo. Incluye artículos, diagramas, opiniones sobre los compañeros y especialmente reflexiones personales sobre su rol de líder en y vinculación con cambios que desea hacer para que su gestión sea de mejor calidad. En algunos casos los participantes vinculan las barreras para liderar en el ambiente profesional con sus procesos personales. Así se expresa en la siguiente cita:

(...) a mis 50 años, cumplidos hace muy poco, con 26 años de experiencia como docente de aula los primeros diez, y hasta marzo pasado, formando parte del equipo directivo de un colegio, debo aceptar que me cuesta reconocer que hay situaciones que no sé manejar; mi capacidad de concentración y centramiento en lo emocional me han jugado malas pasadas, desde mi vida personal hasta laboral. Me cuesta escuchar a las personas y no es por falta de interés, o así lo creía, sino que, por no saber escucharme a mí misma, difícilmente lo podía manejar o dominar. 
Con las reflexiones realizadas mensualmente se elabora un ensayo en el que analizan su liderazgo y se autogeneran desafíos.

3. Taller de escritura. Es una actividad grupal en la que los participantes desarrollan pequeñas narrativas autobiográficas sobre las tres políticas públicas comentadas anteriormente. Después de que cada participante finaliza su texto lo socializa en voz alta, iniciando el intercambio de comentarios. Se incentiva así la interacción oral y escrita entre los participantes.

El que narra selecciona, relata y da fuerza interpretativa a recuerdos específicos [...] Las narrativas autobiográficas se vuelven más complejas por el contexto en el que son producidas para quien o con quien se construye el relato, el momento y la situación en que se da la elaboración, y el propósito (Lapadat, 2009, p. 958).

\section{Resultados}

\subsection{Alta Dirección Pública}

De los ciclos de vida desarrollados, el camino para ser director es diferente entre los colegios particulares subvencionados de congregaciones religiosas y los municipales. En los primeros se trata de una posición que requiere la confianza de la congregación religiosa sostenedora y se accede por invitación a participar o por ser religioso. En los otros dos casos los inspectores llegan al cargo por contar con trayectoria en el establecimiento, pero en el momento de ocupar subrogancias de director el cargo es concursado discretamente y entregado a alguien de afuera, como se evidencia en el siguiente relato:

A fines del año 2015 llegaron los rumores de que se cambiaba de dirección y que sería uno laico. En mi mente creí que iba a ser llamada a serlo... pero era distinto a lo que pensaba... se había realizado un concurso y yo no lo supe... por tanto nadie del colegio postuló a él... llegó el 2015 y se presenta el nuevo director, que para mi sorpresa era conocido, pero igual volvieron los miedos... ¿Qué iba a realizar? ¿Cuáles serían mis funciones y cuál sería mi rol dentro del colegio? ¿Habrá despidos?

En el caso de los establecimientos municipales, con la aprobación de la ley de la Alta Dirección Pública se evidencian diversas prácticas, resultados y opiniones. Por una parte los sostenedores consideran que los concursos deben ser ganados por las personas que los ejercen, por lo que incentivan la participación de los directivos docentes con el objetivo de bloquear la participación de personas de fuera de la comuna, como se manifiesta en el siguiente comentario:

En mi caso personal, yo participé del primer concurso realizado en la comuna por petición que hizo el sostenedor a todos los equipos de gestión escolar, dado que él temía la participación de externos, ya que podía dejar fuera a gente de la comuna. Manifestaba que era triste que quedara fuera la persona que lo ejercía, por lo que había que participar, lo que me quedaba claro que el espíritu de la ley no comulgaba con la política comunal de mantener el statu quo.

Otra alternativa fue declarar los concursos desiertos, como se señala en la siguiente cita:

Cuando llegamos los tres ese día a la presentación [se refiere a los seleccionados después de las entrevista], se nos recibió en la Dirección de Educación de la Corporación Municipal y nos comunicaron que al concurso se le había dado término y había quedado desierto. La sorpresa fue mayúscula. Interiormente lo consideré una falta de respeto, pero como único representante de la Corporación no discutí. Los otros compañeros sí, y la explicación que nos dieron fue que dadas las inquietudes políticas de los jóvenes del momento y las continuas marchas, protestas y tomas, no correspondíamos al perfil que requería la institución.

Desde la perspectiva de los narradores, la Alta Dirección Publica era también una amenaza para los sostenedores. Se temía que llegaran postulantes de fuera de la comuna con otras habilidades y se conformaran ternas en que los tres candidatos no reunieran el perfil político que la comuna requería. Por lo que una vía de solución fue declarar desierto el concurso y nombrar a un director subrogante que podía ejercer el cargo por cinco años. 
La pérdida de los concursos de alta dirección evidencia una situación que marca la vida profesional y personal de los directivos, como indica uno de los entrevistados:

En mi caso fue la peor experiencia, ya que las bases dejaron sin puntaje mi currículum, mi evaluación psicolaboral y el mérito al que alude la ley de ADP, no fue tomado en cuenta. Decidieron tres personas en forma subjetiva en una entrevista de no más de 40 minutos.

En consecuencia, el siguiente comentario presenta la opinión de los directivos que ven el proceso desde fuera:

Le tengo miedo, porque mi carrera no ha sido convencional y porque considero que está teñido políticamente. Reconozco, además, que no me agrada entrar a competir por sobrevivir y visceralmente así lo veo, como una lucha por sobrevivir, donde se impone el más fuerte, el que tiene las mejores conexiones, donde la gente miente y se defiende para asegurar un puesto en función de su remuneración más que de su vocación.

\subsection{Evaluación docente}

La obligatoriedad de la evaluación para los docentes del sistema municipal es de alto impacto. En los casos de dos evaluaciones deficientes consecutivas el profesor es indemnizado y desvinculado. Las siguientes citas reflejan la situación que vivían los directivos docentes al inicio de la implementación.

Mi función, entonces, era apoyar, darles tiempos dentro de la jornada (por ejemplo, horas de consejo que ellos podían usar para preparar portafolio). Por supuesto, el agobio, la angustia, el cansancio y la incertidumbre para los profesores es un lugar común.

El momento era muy difícil, los directivos docentes no contaban con formación específica para apoyar a los docentes que serían evaluados, y la información que se les hacía llegar se relacionaba con los procesos de implementación y no con aspectos pedagógicos que ellos necesitaban. Se sentían apoyando un proceso que desconocían y con mucho temor de cometer errores que pudieran perjudicar a algún docente. La ambigüedad estaba presente, se adueñaba de la agenda de trabajo de los colegios entre agosto y octubre, como evidencia el siguiente comentario:

En mi rol de UTP no sabía cuáles eran mis obligaciones, pues por un lado me decían que no debía intervenir mucho porque si les iba mal me culparían de una mala asesoría y, si no lo hacía pensaba yo-, podían enrostrarme poca preocupación. Así que tomé una decisión personal, que me costó un desgaste más, pero a la vez una fuerte compenetración y validación de mi rol como UTP y como compañera de trabajo en la escuela. Al principio esperé a que se acercaran con sus dudas y desde allí, todos los años y con apoyo de la Dirección, he contribuido en lo que puedo a sus inquietudes. Siempre he sentido que es peligroso, hace un tiempo me daba miedo, así que con los que me llevaba mejor y había afinidad me involucré más, y a los más lejanos finalmente los tuve cercanos aunque fuera por este proceso.

La implementación de la evaluación docente también es vista como un proceso negativo, como se señala en la siguiente narración:

Lamentablemente, desde el comienzo se tiñó este proceso con la política y los gremios, y se alargó un tiempo en llevarlo a efecto. Lo que más me dolía era que aquellos más deficitarios se resguardaban en las posiciones que eran más contrarias a la Evaluación Docente y transmitían un clima de temor a todo el cuerpo docente, señalando que era de carácter punitiva. Luego de que pasó la gran resistencia, me pareció muy engañoso y poco ético que una alta proporción de profesores mandara a hacer los portafolios y los pagasen, que eligieran el mejor curso para la filmación, o que ofrecieran alguna actividad a posteriori a los alumnos, una vez finalizada la película, para que se comportaran adecuadamente tranquilos y atentos, transformándose la actividad evaluativa en un show falso, con la entrega posterior de un surtido de completos, tortas, onces, etc., que compartían con los mismos profesores una vez que se iban los evaluadores. 
La controversia política sobre la evaluación docente se tradujo en rencillas entre los profesores, los partidarios y los que no querían su implementación, lo que afectaba el clima y convivencia entre los profesores. Debido al impacto profesional y personal de la evaluación docente los municipios y directores implementaban acciones de apoyo a la preparación de los portafolios de los profesores, en parte porque paulatinamente éstos fueron asociados a la calidad de los aprendizajes de los alumnos. Ello, unido a las estrategias de difusión de los resultados de pruebas estandarizadas, presionaba a los directores a comprometerse con el logro de los docentes.

En el caso de los colegios particulares subvencionados cada sostenedor puede o no tener procesos de evaluación docente. De acuerdo a las experiencias contadas, aquí los docentes también presentan resistencia, la que es controlada de dos formas: la primera con la participación del sindicato en la definición de criterios de evaluación y sus efectos, y la segunda a través del apoyo de un asesor externo que facilita el proceso y lo va desarrollando en forma colaborativa. En ambos casos el clima organizacional es identificado como un aspecto clave para el éxito de la evaluación. La siguiente cita ilustra este aspecto:

Ahora me gustaría mucho que mis profesores lo tuvieran [se refiere al sistema de evaluación], pero no es así y creo que costará implementarlo, dada las resistencias y la larga trayectoria que tienen los antiguos sin haberse jamás enfrentado a una evaluación formal. De hecho siento una cultura muy reactiva a la retroalimentación directa. La gente tiene miedo del juicio externo porque han vivido desde el castigo. Eso me da pena porque no es mi experiencia.

\subsection{Ley de Subvención Preferencial}

Esta ley ha implicado para los equipos directivos desarrollar planes de mejora en los que se comprometen a lograr ciertas metas y a utilizar los recursos financieros acordes a ella y a las actividades propuestas. El siguiente relato muestra los desafíos que significó la implementación de la Ley SEP.

La Ley SEP, que conlleva el Plan de Mejoramiento Educativo [PME], trajo a mi vida profesional muchas oportunidades tanto personales como a la organización. A través de ella aprendí a elaborar un PME. Al primero lo llamamos "Frankenstein" porque era monstruoso en su tamaño y también por el agotador proceso de tomar algo que nos lanzaron sin mayor preparación. Alcancé a formular siete PME. Complicado siempre fue hacer que toda la comunidad participara en su elaboración, la gestión de los tiempos no era la más adecuada, además todos estábamos aprendiendo, incluso desde la DEPROV, nuestros asesores estaban aprendiendo y guiando a la vez. Para mí, como directora, existía la presión de cumplir las metas, hacer calzar los recursos, desarrollar las acciones de las cuatro áreas del PME y darme cuenta objetivamente de la vulnerabilidad de mis alumnos.

Uno de los aspectos destacados por los directivos es que ha sido muy importante contar con recursos y utilizarlos de forma autónoma, acorde a las necesidades de las comunidades educativas. También ha sido un aprendizaje importante utilizar datos para tomar decisiones, el comentario de uno de los entrevistados es el siguiente:

¡Pero no se preocupen! ¡Porque todos estos cambios vienen con plata!, nos decían supervisores y sostenedores; ега en el fondo lo mismo pero con recursos, más tensión, más exigencias. Al final nos sirvió para aprender a trabajar con datos, a incluir a toda la comunidad educativa en la toma de decisiones, a atreverse a recibir asesorías de externos que nos ponen el dedo en la llaga de la gran pregunta que siempre nos ha movido el piso hasta hoy.

¿Por qué razón teniendo recursos, movilizando estrategias, realizando acciones para mejorar los resultados educativos, estos siguen siendo fluctuantes y no ascendentes? La falta de experiencia de los directivos docentes en el manejo de recursos ha implicado dificultades y en algunos casos responder a sanciones monetarias por el uso de los recursos en actividades que no debían de financiarse con recursos de la Ley SEP, como evidencia en el siguiente relato:

Al principio sólo sabía que los dineros se destinaban para la adquisición de materiales de los aprendizajes de los estudiantes y que se podían cancelar algunas horas de los profesores por concepto SEP, pero al parecer los ingresos eran mayores, y producto [de eso] -no sé si del 
desconocimiento u otra razón- estos recursos fueron mal utilizados y en el 2012 hubo una gran diferencia en la declaración, por tanto tuvimos una multa que cancelar.

Esto ha implicado aprendizajes en los procesos de asignación de recursos, como se indica en el siguiente comentario:

Hoy en día se trabajan estos recursos con listados de adquisición corroborando en qué se destinan de acuerdo al PME, a sus acciones y objetivos. El director y yo hemos revisado concienzudamente estos datos y conocemos los recursos que se destinan, ya que hemos ingresado a la gratuidad y, por ende, recibimos más dineros por concepto SEP 1, SEP 2 y gratuidad.

\section{Conclusiones}

Desde una aproximación autoetnográfica, este estudio proporciona evidencia sobre cómo un grupo de directivos docentes senior ha vivenciado políticas educativas de alto impacto, tanto para ellos, como para sus docentes y la escuela. Uno de los primeros aspectos a destacar es que las implementaciones iniciales de los cambios les despiertan incertidumbre y temor. Perciben que la única forma es aprender haciendo, lo que implica miedo a equivocarse y perjudicar, por ejemplo, a un docente en su evaluación, o a las finanzas de la institución, que puede ser multada por la Superintendencia de Educación. Esta sensación de incertidumbre tiene dos formas de expresión: involucrarse activamente adquiriendo nuevos aprendizajes, como lo especifica una directora: "Fui a cuanta capacitación pude. Tenía que entender cómo era la evaluación docente", o sólo formando parte de aquellas tareas más directas del cargo.

El rol de un directivo docente implica estar siempre atento a los cambios externos y tratar de comprender o anticiparse a ellos, generar estrategias de respuestas rápidas y tranquilizadoras para los docentes. Las orientaciones que reciben de las autoridades ministeriales inicialmente son avanzar e implementar las acciones, sin tiempo para reflexionar, por lo que en muchas oportunidades sienten temor y ven los cambios como una amenaza. Esto ocurre especialmente con aquellos que están próximos a jubilarse, los que prefieren ser más cautelosos en sus relaciones.

También llama la atención que los directivos no mencionan a sus equipos de trabajo. Asumen que las soluciones dependen de ellos y que los integrantes de los equipos implementan sus iniciativas. Se sienten muy responsables de todos los resultados, lo que genera agobio emocional.

Este grupo de directivos senior se acerca a su jubilación y uno de los aspectos que les produce mayor incertidumbre y sentimientos negativos es el modelo de selectividad de los cargos a través de la Alta Dirección Pública. No se sienten capacitados para ejercer la influencia política con los alcances para mantener sus cargos. Más aún, temen a los cambios radicales, como sería la creación de la Agencia de la Calidad.

Los resultados indican que los directivos docentes son los implementadores de las políticas públicas, que se encuentran en una posición estratégica para comprender las tensiones que éstas producen en ellos mismos y en sus comunidades. Mayor capacitación y comprensión de las políticas ayuda a disminuir los conflictos profesionales y personales que éstas provocan. Esta oportunidad de poder entender los procesos desde una mirada centrada en lo vivencial es una fortaleza que ofrece la autoetnografía como finalidad y método. Es por esto que se considera relevante comprender las políticas públicas desde una mirada fenómeno-persona, sin tener que ser por ello una mirada reduccionista. De este modo, es posible advertir una correlación entre procesos e individuos, contextos y realidades personales, correlación que debe ser reposicionada en términos educativos.

\section{Referencias}

Anderson, L. (2006). Analytic autoethnography. Journal of Contemporary Ethnography, 35(4), 373-395. doi:10.1177/0891241605280449

Austin, J. y Hickey, A. (2007). Autoethnography and teacher development. The International Journal of Interdisciplinary Social Sciences, 2. Recuperado de https://eprints.usq.edu.au/3287/ 
Blinne, K. (2014). Writing my life: a narrative and poetic-based autoethnography. Journal of Poetry Therapy, 23(3), 183-190.

Bravo, D., Falck, D., González, R., Manzi, J. y Peirano, C. (2008). La relación entre la evaluación docente y el rendimiento de los alumnos: evidencia para el caso de Chile. Recuperado de http://eoepsabi.educa.aragon.es/descargas/H Recursos/h 1 Psicol Educacion/h 1.4.Eval dese mp docente/1.5. Relacion evaluac doc rendim.pdf

Bruner, E. (1997). Ethnography as narrative. En L. Hinchman y S. Hinchman (Eds.), Memory, identity, community: The idea of narrative in the human sciences (pp. 264-280). New York State University.

Chang, H. (2008). Autoethnography as method. Walnut Creek, CA: Left Coast Press.

Chang, H., Longman, K. y Franco, M. (2014) Leadership development through mentoring in higher education: A collaborative autoethnography of leaders of color. Mentoring \& Tutoring: Partnership in Learning, 22(4), 373-389.

Curran, J. (2012). An autoethnography of transition. Symposium: Transition issues and persons with ID. Journal of Intellectual Disability Research, 56(7-8). doi:10.1111/j.1365-2788.2012.01583_8.x

Day, C. y Gu, Q. (2012). Profesores: vidas nuevas, verdades antiguas: una influencia decisiva en la vida de los alumnos. Madrid, España: Narcea.

Ellis, C. (2009). Fighting back or moving on: An autoethnographic response to critics. International Review of Qualitative Research, 2(3), 371-378.

Ellis, C. y Bochner, A. (2000). Autoethnography, personal narrative, reflexivity. Researcher as Subject. En N. Denzin y Y. Lincoln (Eds.), Handbook of qualitative research (pp. 733-768). Thousand Oaks, CA: Sage.

Flanagan, J. (1954). The critical incident technique. Psychological Bulletin, 51(4), 327-358. Recuperado de https://www.apa.org/pubs/databases/psycinfo/cit-article.pdf

Hamilton, L. y Corbett-Whittier, C. (2012). Using case study in education research. Londres: Sage.

Holt, N. L. (2003). Representation, legitimation, and autoethnography: An autoethnographic writing story. International Journal of Qualitative Methods, 2(1). Recuperado de

https://sites.ualberta.ca/ iiqm/backissues/2 1/html/holt.html

Humphreys, M. (2005). Getting personal: Reflexivity and autoethnographic vignettes. Qualitative Inquiry, 11(6), 840-860. doi:10.1177/1077800404269425

Lapadat, J. (2009). Writing our way into shared understanding: Collaborative autobiographical writing in the qualitative methods class. Qualitative Inquiry, 15(6), 955-979. doi:10.1177/1077800409334185

Legee, M. (2014). Autoethnography and teacher education: Snapshot stories of cultural encounter. Australian Journal of Teacher Education, 39(5), 117-134.

Maxwell, J. (2005). Qualitative research design: An interactive approach. Thousand Oaks, CA: Sage.

Ministerio de Educación. (2008). Leyes de subvención preferencial. Chile: Autor.

Patton, M. (2001). Qualitative research and evaluation methods. Thousand Oaks, CA: Sage.

Polkinghorne, D. (1995). Narrative configuration in qualitative analysis. En J. Hatch y R. Wisniewski (Eds.), Life history and narrative (pp.5-23). Londres: Falmer Press.

Raczynski, D., Muñoz, G., Weinstein, J. y Pascual, J. (2016). Subvención escolar preferencial (SEP) en Chile: Un intento por equilibrar la macro y micro política escolar. Revista Iberoamericana sobre Calidad, Eficacia y Cambio en Educación, 11(2).

Reed-Denahay, D. (2009) Anthropologists, education, and autoethnography. Reviews in Anthropology, 38(1), 28-47.

Sun, Y., Correa, M., Zapata, A. y Carrasco, D. (2011). Resultados: qué dice la evaluación docente acerca de la enseñanza en Chile. En J. Manzi, R. González y Y. Sun (Eds.), La evaluación docente en Chile (pp. 91-136). Chile: Facultad de Ciencias Sociales /Mide uc. Recuperado de 


\section{http://www.mideuc.cl/libroed/pdf/La Evaluacion Docente en Chile.pdf}

Vasconcelos, E. (2011). I can see you: An autoethnography of my teacher-student self. The Qualitative Report, 16(2), 415-440.

Wall, S. (2006). An autoethnography on learning about ethnography. International Journal of Qualitative Methods, 5(2), 1-12. Recuperado de https://sites.ualberta.ca/ iiqm/backissues/5 2/PDF/wall.pdf

Wolcott, H. (2008). Ethnography: A way of seeing (2a. ed.). Nueva York: Alta Mira. 